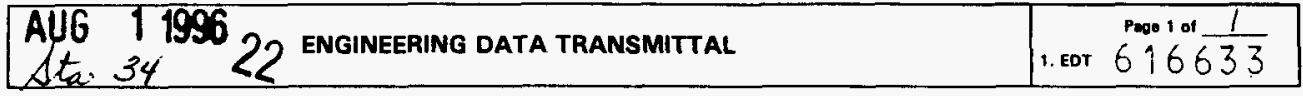

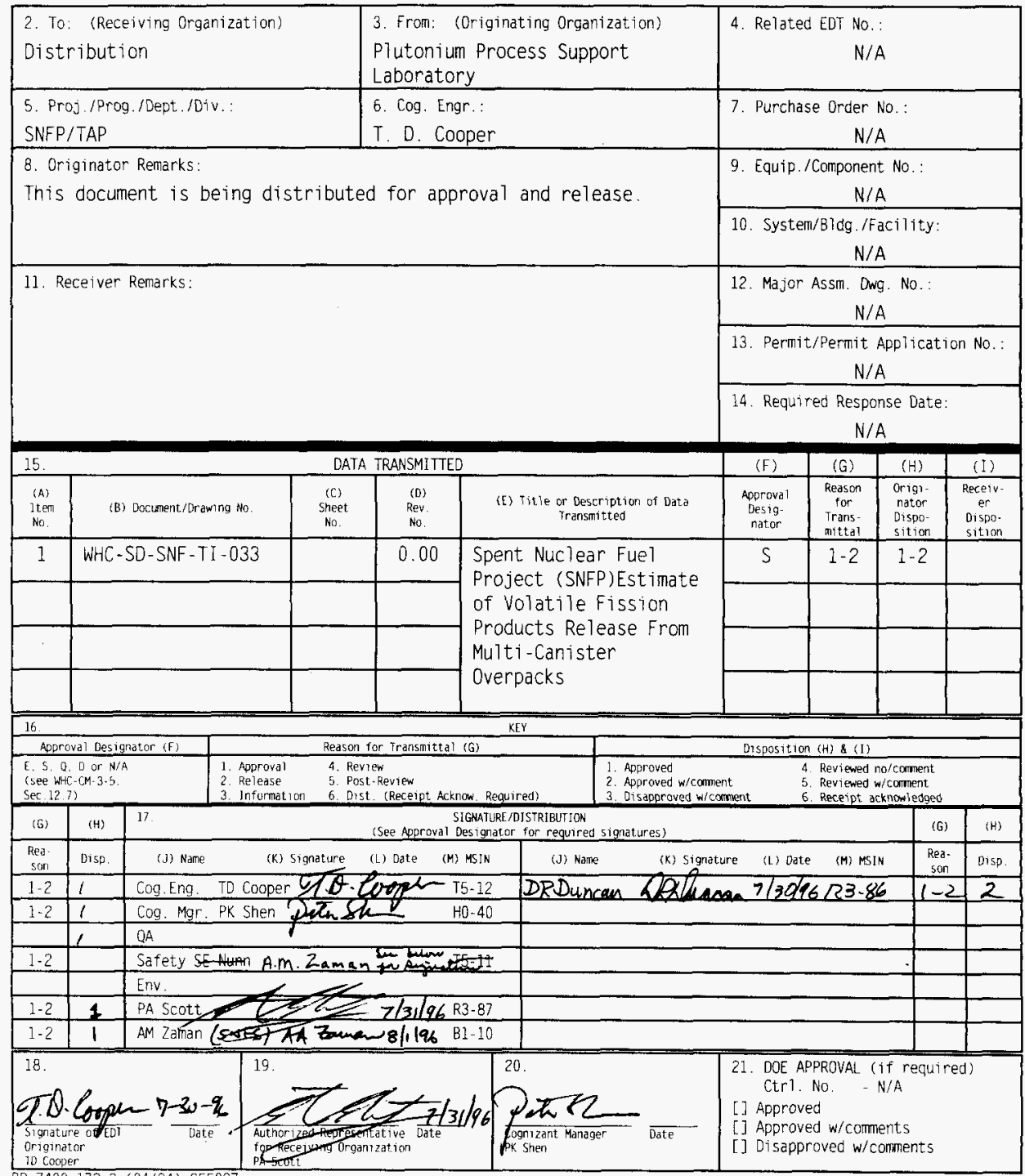

BD-7400-172-2 (04/94) GEF097 
WHC-SD-SNF-TI-033, Rev. 0

\title{
Spent Nuclear Fuel Project \\ Estimate of Volatile Fission Products \\ Release From Multi-Canister Overpacks
}

\author{
Thurman D. Cooper \\ Westinghouse Hanford Company, Richland, WA 99352 \\ U.S. Department of Energy Contract DE-AC06-87RL10930 \\ EDT/ECN: 616633 \\ UC: 510 \\ Org Code: 15500 \\ Charge Code: LB040 \\ B\&R Code: EW3135040 \\ Total Pages: $2225 w_{-1-96}$
}

Key Words: MCO, cold vacuum dried, hot vacuum dried, Canister Storage Building, fission

Abstract

Spent N-Reactor fuel will be moved from wet pool storage to dry storage al Hanford Washington This fuel will be sequentially loaded into a Multiple Container Overpack (MCO), moved to the cold vacuum drying station, drained, cold vacuum dried, shipped to the Canister Storage Building (CSB), staged for up to 2 years, hot vacuum dried at $300^{\circ} \mathrm{C}$, hot conditioned at $150^{\circ} \mathrm{C}$, and finally, sealed and stored for up to 75 years in the CSB

During each proposed process step, the volatile radioactive fission products released to the atmosphere were estimated Thtium is the only volatite tission product released in significant amounis during each process step

For an aceident scenaro involving interior MCO lemperatures of $600^{\circ} \mathrm{C}$ for up to 8 hours, it was estimated that many volatile fission products are released

TRADEMARK DISCLAIMER. Reference herein to any specific commercial product, process, or service by trade name, uademark, manufacturer, or otherwise, does not necessarily constitute or imply its endorsement, recommendation, or favoring by the United States Govemment or any agency thereof or its contractors or subcontractors.

Printed in the United States of America. To obtain copies of this document, contact: WHC/BCS Document Control Senvices, PO Box 1970, Mailstop H6-08, Richland WA 99352, Phone (509) 372-2420; Fax (509) 376-4989

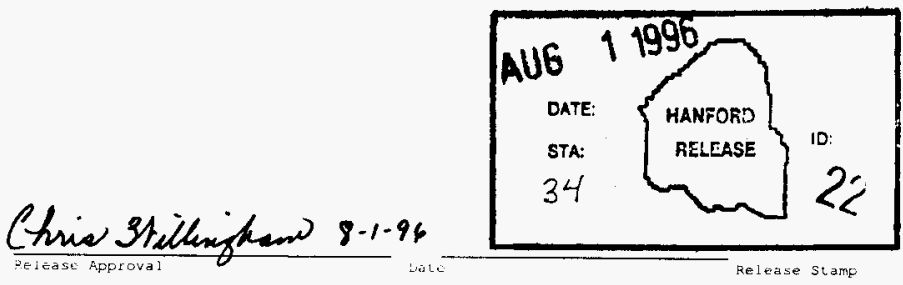




\section{SPENT NUCLEAR FUEL PROJECT ESTIMATE OF VOLATILE FISSION PRODUCTS RELEASE FROM MULTI-CANISTER OVERPACKS}

July 1996

Prepared for the U.S. Department of Energy

Office of Environmental Restoration and Waste Management

Westinghouse Hanford Company

P.O. Box 1970

Richland, Washington 
WHC-SD-SNF-TI-033

Revision 0

This page intentionally left blank. 


\section{CONTENTS}

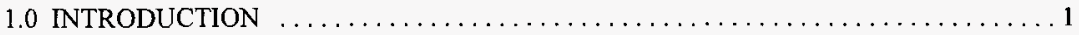

2.0 EMPIRICAL MODELS $\ldots \ldots \ldots \ldots \ldots \ldots \ldots \ldots \ldots \ldots \ldots \ldots \ldots \ldots \ldots \ldots$

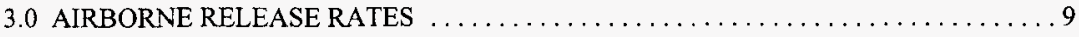

4.0 FISSION PRODUCT RELEASE CASES $\ldots \ldots \ldots \ldots \ldots \ldots \ldots \ldots \ldots \ldots \ldots \ldots \ldots \ldots \ldots \ldots$

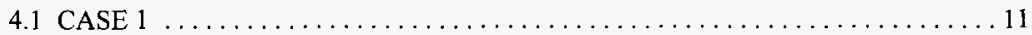

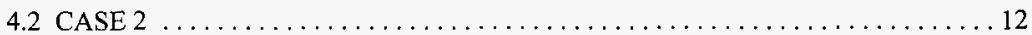

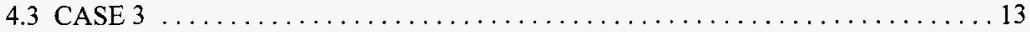

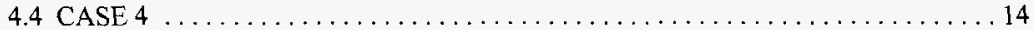

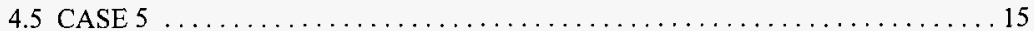

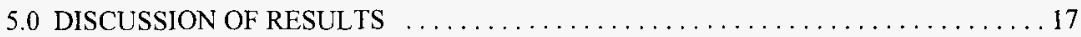

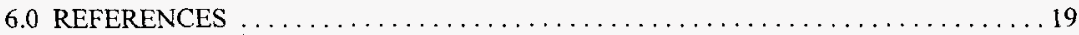




\section{LIST OF TABLES}

1. Parameters for Radionuclide Loss from $\mathrm{UO}_{2}$ Matrix $\ldots \ldots \ldots \ldots \ldots \ldots \ldots \ldots$

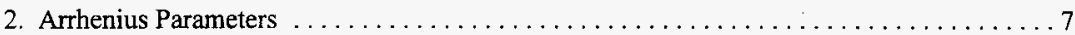

3. High Temperature Fission Product Release from Uranium Metal $\ldots \ldots \ldots \ldots \ldots \ldots \ldots 1$

4. Fission Product Release During Hot Vacuum Drying $\ldots \ldots \ldots \ldots \ldots \ldots \ldots \ldots \ldots$

5. Fission Product Release During Cold Vacuum Drying $\ldots \ldots \ldots \ldots \ldots \ldots \ldots$

6. Fission Product Release During Hot Conditioning $\ldots \ldots \ldots \ldots \ldots \ldots \ldots \ldots \ldots$

7. Fission Product Release During Long Term Storage $\ldots \ldots \ldots \ldots \ldots \ldots \ldots \ldots \ldots$

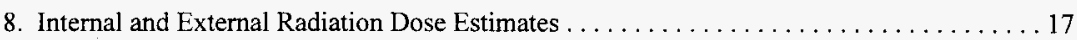

\section{LIST OF FIGURES}

1. Diffusion Rate Constants for Strontium $/ Y t t r i u m-90 \ldots \ldots \ldots \ldots \ldots \ldots \ldots$ 


\section{SPENT NUCLEAR FUEL PROJECT ESTIMATE OF VOLATILE FISSION PRODUCTS RELEASE FROM MULTI-CANISTER OVERPACKS}

\subsection{INTRODUCTION}

Spent nuclear fuel (SNF) has been stored at Hanford in the $\mathrm{K}$ Basin pools for up to 30 years. The SNF will be moved from the K Basins to a dry, interim storage facility, the Canister Storage Building.

Spent fuel has been irradiated and contains many fission products. Some of these fission products are volatile and are potential environmental hazards. During the drying, stabilization, and storage of the fuel, the environmental risk must be understood and minimized.

As long as the fuel is intact and non-oxidized, the fission products are contained within the metal matrix. Release of the fission products through solid state diffusion is extremely low at ambient temperature.

Mechanisms for increasing the fission product release rates include heating the fuel and oxidation of the fuel. Heating the fuel increases the solid state diffusion rate. A 50-100 micron layer of oxide on the uranium metal surface is formed apon contact with either water or oxygen. Diffusion of fission products through uranium oxide is much faster than diffusion through the metal, therefore, oxidation is the key mechanism for fission product release. 
WHC-SD-SNF-TI-033

Revision 0

This page intentionally left blank. 


\subsection{EMPIRICAL MODELS}

It must be clearly stated that no data is available for the release of fission products from either uranium metal or oxide in the temperature range of ambient to $300{ }^{\circ} \mathrm{C}$ (the range of interest). Data does exist for the release of fission products from uranium dioxide in the temperature range $1000-2200^{\circ} \mathrm{C}$ from the Light Water Reactor (LWR) severe accident studies (Owczarski 1996). Two empirical models have been formulated for this higher temperature range.

The empirical models initially used in the LWR severe accident studies are based on the definition of a fractional release of the radionuclide $r$ per unit time, $\mathrm{k}_{\mathrm{r}}$, time-1. This $\mathrm{k}_{\mathrm{f}}$ is defined as the fraction of $r$ released to the atmosphere per unit of time from the mass of $r, \mathrm{~m}_{\mathrm{r}}$, in a given amount of $\mathrm{UO}_{2}$.

$$
\mathrm{dm}_{\mathrm{r}} / \mathrm{dt}=-\mathrm{k}_{\mathrm{r}} \mathrm{m}_{\mathrm{t}}
$$

This rate constant is a function of temperature alone. Equation (1) was applied to all positions in the LWR during a severe accident scenario (loss of coolant, heat up, and melting), covering a large range of temperatures (NRC 1981). The equation(s) used for calculating the Ks is an empirical fit of data of the form:

$$
\mathbf{k}_{\mathrm{r}}=\mathrm{A}_{\mathrm{r}} \exp \left(\mathrm{B}_{\mathrm{s}} \mathrm{T}_{\mathrm{c}}\right)
$$

where $T_{c}$ is the oxide temperature in degrees Celsius.

Values used for the As and Bs are found in Table 1. They are taken from NRC 1981 (see next paragraph) for the low temperature range $1000-2200{ }^{\circ} \mathrm{C}$. Because they are a function of temperature alone, they are independent of exposed surface area and degree of cladding failure. Using this approach in an $\mathrm{MCO}$ only requires that the quantities of $\mathrm{UO}_{2}$ present and the temperature histories of each portion need to be tracked in an MCO model.

Since a number of radionuclides are not specifically identified in NRC 1981, but Table 1 is assigned values of the As and Bs for similar elements. All of the transuranic elements were assigned fuel $\mathrm{U}$ values. Iron, nickel, and cobalt were assigned the structure (steel) values. 
Table 1. Parameters for Radionuclide Loss from $\mathrm{UO}_{2}$ Matrix (2 Sheets).

\begin{tabular}{|c|c|c|c|c|}
\hline \multirow[b]{2}{*}{ Isotope } & \multicolumn{2}{|c|}{$k=A \exp \left(B t_{c}\right)$} & \multicolumn{2}{|c|}{$\mathrm{K}^{\prime}=\mathbf{k}_{e} \exp \left(\mathrm{Q} / \mathbf{R T} \mathrm{T}_{\psi}\right)$} \\
\hline & $A, 1 / \min$ & $B, 1 T_{c}$ & $\mathrm{~K}_{0}, 1 / \mathrm{min}$ & $\mathrm{Q}, \mathrm{kJ} / \mathrm{mol}$. \\
\hline $\mathrm{Pu}-241$ & $1.4 \mathrm{E}-14$ & $6.77 \mathrm{E}-03$ & & \\
\hline Am-241 & $1.4 \mathrm{E}-14$ & $6.77 \mathrm{E}-03$ & & \\
\hline Pu-239 & $1.4 \mathrm{E}-14$ & $6.77 \mathrm{E}-03$ & & \\
\hline $\mathrm{Pu}-238$ & $1.4 \mathrm{E}-14$ & $6.77 \mathrm{E}-03$ & & \\
\hline Pu-240 & $1.4 \mathrm{E}-14$ & $6.77 \mathrm{E}-03$ & & \\
\hline $\mathrm{Sr} / \mathrm{Y}-90$ & $7.28 \mathrm{E}-11$ & $6.77 \mathrm{E}-03$ & $1.20 \mathrm{E}+00$ & 200 \\
\hline $\mathrm{Cm}-244$ & $1.40 \mathrm{E}-14$ & $6.77 \mathrm{E}-03$ & & \\
\hline $\mathrm{Ru} / \mathrm{Rh} \mathrm{m}-106$ & $1.38 \mathrm{E}-08$ & $7.68 \mathrm{E}-03$ & $1.90 \mathrm{E}+09$ & 710 \\
\hline $\mathrm{Cs} / \mathrm{Ba} \mathrm{m}-137$ & $1.65 \mathrm{E}-07$ & $6.77 \mathrm{E}-03$ & $8.50 \mathrm{E}+05$ & 292 \\
\hline $\mathrm{Pm}-147$ & & & $1.90 \mathrm{E}+09$ & 710 \\
\hline $\mathrm{Ce} / \mathrm{Pr}-144$ & $1.6 \mathrm{E}-12$ & $6.77 \mathrm{E}-03$ & $5.20 \mathrm{E}+11$ & 852 \\
\hline Eu-154 & $1.00 \mathrm{E}-08$ & $6.77 \mathrm{E}-03$ & & \\
\hline U-234 & $1.4 \mathrm{E}-14$ & $6.77 \mathrm{E}-03$ & & \\
\hline $\mathrm{Cs}-134$ & $1.65 \mathrm{E}-07$ & $6.77 \mathrm{E}-03$ & $8.50 \mathrm{E}+05$ & 292 \\
\hline $\mathrm{Pu}-242$ & $1.4 \mathrm{E}-14$ & $6.77 \mathrm{E}-03$ & & \\
\hline $\mathrm{Sb} / \mathrm{Te} \mathrm{m}-125$ & & & $2.62 E+02$ & 207 \\
\hline $\mathrm{Cd}-113 \mathrm{~m}$ & & & $1.90 \mathrm{E}+09$ & 710 \\
\hline Sm-151 & & & $5.20 \mathrm{E}+11$ & 852 \\
\hline Eu-155 & $1.00 \mathrm{E}-08$ & $6.77 \mathrm{E}-03$ & & \\
\hline Co-60 & $3.20 \mathrm{E}-11$ & $5.76 \mathrm{E}-03$ & & \\
\hline Eu-152 & $1.00 \mathrm{E}-08$ & $6.77 \mathrm{E}-03$ & & \\
\hline I-129 & & & $1.90 \mathrm{E}+07$ & 335 \\
\hline $\mathrm{Kr}-85$ & & & $3.20 \mathrm{E}+04$ & 335 \\
\hline $\mathrm{Fe}-55$ & $3.20 \mathrm{E}-11$ & $5.76 \mathrm{E}-03$ & & \\
\hline
\end{tabular}


Table 1. Parameters for Radionuclide Loss from $\mathrm{UO}_{2}$ Matrix (2 Sheets).

\begin{tabular}{|c|c|c|c|c|}
\hline \multirow[b]{2}{*}{ Isotope } & \multicolumn{2}{|c|}{$k=A \exp \left(B t_{c}\right)$} & \multicolumn{2}{|c|}{$K^{\prime}=k_{0} \exp \left(Q / R T_{k}\right)$} \\
\hline & $\mathrm{A}, 1 / \mathrm{min}$ & $\mathbf{B}, 1 \mathrm{~T}_{\boldsymbol{c}}$ & $\mathbf{K}_{p}, 1 / \mathbf{m i n}$ & $\mathbf{Q}, \mathbf{k} \mathbf{J} / \mathbf{m o l}$ \\
\hline $\mathrm{Ni}-63$ & $3.20 \mathrm{E}-11$ & $5.76 \mathrm{E}-03$ & & \\
\hline Tc-99 & & & $1.90 \mathrm{E}+09$ & 710 \\
\hline Se-79 & & & $1.20 \mathrm{E}+00$ & 200 \\
\hline $\mathrm{H}-3$ & & & & \\
\hline C- 14 & & & & \\
\hline
\end{tabular}

Kelly et al. (1984) introduced the Arrhenius form of temperature dependence as being "more reasonable" and a better fit with the experimental data. In this form

$$
k_{r}=k_{T 0} \exp \left(-Q_{r} / R T\right)
$$

where $\mathrm{R}$ is the universal gas constant and $\mathrm{T}$ the absolute temperature in Kelvin degrees.

Table 1 also contains the applicable values of the $\mathrm{k}_{\mathrm{ro}} \mathrm{s}$ and $\mathrm{Q}_{\mathrm{r}} \mathrm{s}$ that were published. Figure 1 displays the $\mathrm{ks}$ calculated from Equations 2 and 3 for strontium/yttrium-90.

Figure 1 is typical in form for the ks calculated for the other fission products also. Over the experimentally calibrated range of 1000 to $2200^{\circ} \mathrm{C}$, these two functions are very similar. However, as noted above, Kelly states that the Arrhenius form is more reasonable and fits the experimental data more closely.

It is unfortunate that the process temperatures proposed for the spent fuel lies in the uncalibrated range below $1000^{\circ} \mathrm{C}$. These two functions diverge widely below $1000^{\circ} \mathrm{C}$, and one must choose which to use. Since the Arrhenius function appears superior in scientific basis and experimental fit, it will be the function used to estimate the release rate for volatile fission products from the MCO.

Since all of the pre-exponential factors and activation energies were not published for each of the isotopes in Table 1, values were estimated based upon similar ionic charge to radius ratios and are presented in Table 2. 
Figure 1. Diffusion Rate Constants for Strontium/Yttrium-90.

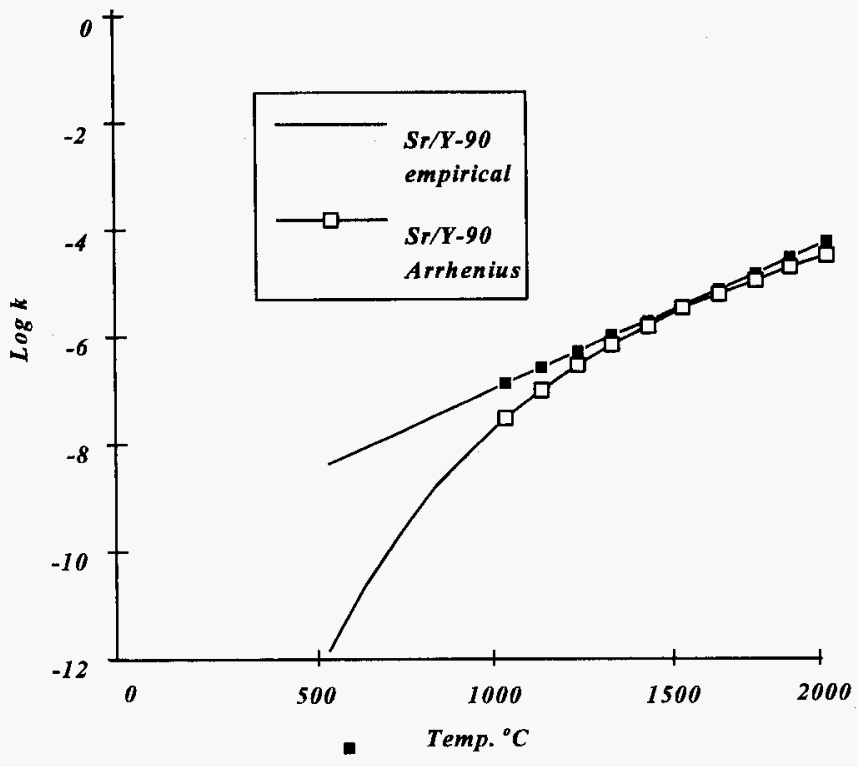


Table 2. Arrhenius Parameters (2 Sheets).

\begin{tabular}{|c|c|c|}
\hline \multirow[b]{2}{*}{ Isotope } & \multicolumn{2}{|c|}{$K^{\prime}=k_{i} \exp \left(Q / R T_{k}\right)$} \\
\hline & $K_{0}, 1 /$ min & Q, kJJ/mol. \\
\hline $\mathrm{Pu}-241$ & $1.90 \mathrm{E}+09$ & 710 \\
\hline Am-241 & $1.90 \mathrm{E}+09$ & 710 \\
\hline Pu-239 & $1.90 \mathrm{E}+09$ & 710 \\
\hline Pu-238 & $1.90 \mathrm{E}+09$ & 710 \\
\hline $\mathrm{Pu}-240$ & $1.90 \mathrm{E}+09$ & 710 \\
\hline $\mathrm{Sr} / \mathrm{Y}-90$ & $1.20 \mathrm{E}+00$ & 200 \\
\hline $\mathrm{Cm}-244$ & $1.90 \mathrm{E}+09$ & 710 \\
\hline $\mathrm{Ru} / \mathrm{Rh} \mathrm{m}-106$ & $1.90 \mathrm{E}+09$ & 710 \\
\hline $\mathrm{Cs} / \mathrm{Ba} \mathrm{m}-137$ & $8.50 \mathrm{E}+05$ & 292 \\
\hline $\mathrm{Pm}-147$ & $1.90 \mathrm{E}+09$ & 710 \\
\hline $\mathrm{Ce} / \mathrm{Pr}-144$ & $5.20 \mathrm{E}+11$ & 852 \\
\hline Eu-154 & $1.90 \mathrm{E}+09$ & 710 \\
\hline $\mathrm{U}-234$ & $1.90 \mathrm{E}+09$ & 710 \\
\hline Cs- 134 & $8.50 \mathrm{E}+05$ & 292 \\
\hline $\mathrm{Pu}-242$ & $1.90 \mathrm{E}+09$ & 710 \\
\hline $\mathrm{Sb} / \mathrm{Te} \mathrm{m}-125$ & $2.62 \mathrm{E}+02$ & 207 \\
\hline $\mathrm{Cd}-113 \mathrm{~m}$ & $1.90 \mathrm{E}+09$ & 710 \\
\hline Sm-151 & $5.20 \mathrm{E}+11$ & 852 \\
\hline Eu-155 & $1.90 \mathrm{E}+09$ & 710 \\
\hline $\mathrm{Co}-60$ & $1.90 \mathrm{E}+09$ & 710 \\
\hline Eu-152 & $1.90 \mathrm{E}+09$ & 710 \\
\hline I-129 & $1.90 \mathrm{E}+07$ & 335 \\
\hline $\mathrm{Kr}-85$ & $3.20 \mathrm{E}+04$ & 242 \\
\hline $\mathrm{Fe}-55$ & $1.90 \mathrm{E}+09$ & 710 \\
\hline $\mathrm{Ni}-63$ & $1.90 \mathrm{E}+09$ & 710 \\
\hline Tc-99 & $1.90 \mathrm{E}+09$ & 710 \\
\hline $\mathrm{Se}-79$ & $1.20 \mathrm{E}+00$ & 200 \\
\hline
\end{tabular}


Table 2. Arrhenius Parameters (2 Sheets).

\begin{tabular}{|c|c|c|}
\hline \multirow{2}{*}{ Isotope } & \multicolumn{2}{|c|}{$K^{\prime}=k_{e} \exp \left(Q / R T_{k}\right)$} \\
\hline & $K_{\mathrm{g}}, 1 / \min$ & Q,kJ/mol. \\
\hline $\mathrm{H}-3^{\prime}$ & & \\
\hline C- $14^{2}$ & $3.20 \mathrm{E}+04$ & 242 \\
\hline
\end{tabular}

1 Monatomic tritium forms tritiated water upon reaction with oxygen. Due to the rapid hydrogen isotope exchange occurring upon water and hydroxide ions, it is postulated that the release of tritium to the atmosphere is essentially instantaneous. Although proof of this statement is not included, it is a conservative assumption.

2 The rate of diffusion of carbon-14 through uranium dioxide is not known or measured. It will probably form carbon monoxide or carbon dioxide in the presence of oxygen or elemental carbon particles in an anoxic atmosphere. To remain conservative, it is assigned the same diffusion rate as the noble gas krypton-85. 


\subsection{AIRBORNE RELEASE RATES}

If $\mathrm{m}_{\mathrm{ro}}$ is defined as the growth rate of isotope $r$ within the oxide due to oxidation of metal, and $k_{r} m_{r}$ is the airborne source term rate, the change in isotope $r$ with respect to time can be given as:

$$
\mathrm{dm} / \mathrm{dt}=\mathrm{m}_{\mathrm{ro}}-\mathrm{k}_{\mathrm{r}} \mathrm{m}_{\mathrm{r}}
$$

This can be rearranged algebraically to give

$$
\mathrm{k}_{\mathrm{r}} \mathrm{m}_{\mathrm{r}} \quad \mathrm{dt}=-\mathrm{dm}_{\mathrm{r}}+\mathrm{m}_{\mathrm{ro}} \mathrm{dt}
$$

and finally:

$$
\int_{k},{ }_{r}{ }_{r} d t=\mathrm{A}_{\mathrm{st}}=\int^{m}{ }_{r o} d t+\int \boldsymbol{C}^{d m} \text {. }
$$

allowing time to vary between 0 and time $t$, and the mass of a particular fission product to vary over that same time interval:

$$
A_{\mathrm{st}}=\mathrm{m}_{\mathrm{ro}} *\left(\mathrm{t}_{2}-\mathrm{t}_{1}\right)-\left(\mathrm{m}_{\mathrm{r} 2}-\mathrm{m}_{\mathrm{r} 1}\right)
$$

Equation 6 can be solved if the change in $m_{r}$ can be calculated. If Equation 4 is rearranged algebraically:

$$
\mathrm{dm}_{\mathrm{r}} /\left(\mathrm{m}_{\mathrm{ro}}-\mathrm{k}_{\mathrm{r}} \mathrm{m}_{\mathrm{r}}\right)=\mathrm{dt}
$$

the integrated solution is:

$$
\left(m_{r 0}-k_{T} m_{r 2}\right) /\left(m_{r o}-k_{r} m_{r 1}\right)=e^{-k r(L-11)}
$$

and after appropriate algebraic manipulation:

$$
\mathrm{m}_{\mathrm{r} 2}=\mathrm{m}_{\mathrm{r} 1} \mathrm{e}^{-k \mathrm{r}(2-\mathrm{t}-1)}+\left(\mathrm{m}_{\mathrm{ro}} / \mathrm{k}_{\mathrm{T}}\right)\left(1-\mathrm{e}^{-\mathrm{kr}(2-12)}\right)
$$

by substitution of Equation 8 into Equation 6 :

$$
\begin{aligned}
& A_{\mathrm{st}}=\mathrm{m}_{\mathrm{ro}}{ }^{*}\left(\mathrm{t}_{2}-\mathrm{t}_{1}\right)+\left(\mathrm{m}_{\mathrm{r} 1}-\mathrm{m}_{\mathrm{r} 1} \mathrm{e}^{-\mathrm{kr}(\mathrm{L}-\mathrm{k}-1)}\right)-\left(\mathrm{m}_{\mathrm{r} 0} / \mathrm{k}_{\mathrm{r}}\right)\left(1-\mathrm{e}^{-\mathrm{k}(\mathrm{r}(2-\mathrm{t})}\right) \\
& =\mathrm{m}_{\mathrm{ro}} *\left(\mathrm{t}_{2}-\mathrm{t}_{1}\right)+\mathrm{m}_{\mathrm{r} 1}\left(1-\mathrm{e}^{-\mathrm{kr}(12-11)}\right)-\left(\mathrm{m}_{\mathrm{ro}} / \mathrm{k}_{\mathrm{f}}\right)\left(1-\mathrm{e}^{-\mathrm{kr}(\mathrm{L}-\mathrm{t})}\right) \\
& =\mathrm{m}_{\mathrm{ro}} *\left(\mathrm{t}_{2}-\mathrm{t}_{1}\right)+\left(\mathrm{m}_{\mathrm{r} 1}-\left(\mathrm{m}_{\mathrm{ro}} / \mathrm{k}_{\mathrm{r}}\right)\right)\left(1-\mathrm{e}^{-k r(12-1)}\right)
\end{aligned}
$$


since for most MCO applications, $k_{t} t<<1$, and the spreadsheet function of $\exp \left(-k_{r} t\right)$ is inaccurate.

Equation 9 can be expanded for small $x$ as $\exp (-x)=1-x+X^{2} / 2$ !, which gives:

$$
A_{s t}=m_{r} k_{T} t^{*}\left(1-k_{T} t / 2\right)+m_{r o} k_{r} t^{2} / 2
$$
used.

Equation 10 is used for spreadsheet calculations if $\mathrm{k}_{\mathrm{r}} \mathrm{t}<0.01$. Otherwise, Equation 9 is 
WHC-SD-SNF-Tl-033

Revision 0

\subsection{FISSION PRODUCT RELEASE CASES}

\subsection{CASE 1}

Table 3 gives the integrated release of fission products in $\mathrm{Bq} / \mathrm{cm}^{2}$ for the oxidation of exposed metal at $600^{\circ} \mathrm{C}$ at 1 hour, 4 hours, and 8 hours.

Table 3. High Temperature Fission Product Release from Uranium Metal.

\begin{tabular}{|c|c|c|c|c|c|c|c|c|c|c|c|}
\hline \multicolumn{4}{|r|}{ 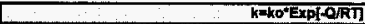 } & \multicolumn{3}{|c|}{ Inpit Temopo ${ }^{\circ} \mathrm{C}$} & & & & & \\
\hline feoliope & CHMTU & Salige & $k 0,1 / m i n$ & Q,kihad. & 0 & $k, 1 /$ & Mro & . Mt: & \multicolumn{3}{|c|}{ interroted De, elocin } \\
\hline & & & & & & & Dotenin ieses & Bqlem & 350 & S4moo & 2800 \\
\hline$P \mathrm{Pu}-241$ & 9450 & $3.50 \mathrm{E}+12$ & $1.90 E+09$ & 710 & 6.357E.34 & $3.814 \mathrm{E}-32$ & 7878315543 & 18889908 & $1.9471 \mathrm{E}-15$ & $3.11532 \mathrm{E}-14$ & $1.246 \mathrm{E}-13$ \\
\hline Am-241 & 142 & $5.25 E+09$ & $1.90 \mathrm{E}+09$ & 710 & 6.357E-34 & $3.814 E-32$ & 118383154 & 253795.443 & $2.9258 \mathrm{BE}-17$ & 4.68123E-16 & $1.672 E-15$ \\
\hline Pu-239 & 139 & $5.14 E+09$ & $1 . \overline{90 E+09}$ & 710 & $6.357 \mathrm{E}-34$ & 3.614E-32 & 115882102 & 248433.567 & $2.864 E-17$ & $4.58233 \mathrm{E}-16$ & 1. $\mathrm{B} 33 \mathrm{E}-15$ \\
\hline $\mathrm{Pu}-238$ & 116 & 4.29E +09 & $1.90 \mathrm{E}+09$ & 710 & $6.357 \mathrm{E}-34$ & $3.814 \mathrm{E}-32$ & 967073654 & 207325.855 & $2.3901 \mathrm{E}-17$ & $3.8241 E-16$ & $1.53 \mathrm{E}-15$ \\
\hline Pu-240 & 95.2 & $3.52 E+09$ & $1.90 E+09$ & 710 & 6.357E-34 & 3.814E-32 & 793667344 & 170150.184 & $19615 \mathrm{E}-17$ & $3.1384 E-16$ & 1.255E-15 \\
\hline $\sin \bar{Y}-90$ & 8480 & $3.14 \mathrm{E}+11$ & 1.2 & 200 & $1,301 \mathrm{E}-12$ & $7.805 \mathrm{E}-11$ & 7089641884 & 15156234.9 & 3575751.89 & 57211979.2 & 228647863 \\
\hline $\mathrm{Cm}+244$ & 26.8 & $9.92 \mathrm{E}+0 \mathrm{~B}$ & $1.90 E+09$ & 710 & $6.357 \mathrm{E}-34$ & $3.814 \mathrm{E}-32$ & 22342736.1 & 47999.4216 & $5.5210 E-18$ & 8.83489E-17 & $3.534 \bar{E}-16$ \\
\hline RuRh m- 906 & 2430 & B. $99 \mathrm{E}+10$ & 1900000000 & 710 & $6.357 \mathrm{E}-34$ & 3.814E.32 & 2025852568 & 4343119.2 & $5.0068 E-16$ & $8.01083 E-15$ & $3.204 \mathrm{E}-14$ \\
\hline Cs/Ba m- 137 & 10600 & $3.92 E+11$ & 850000 & 292 & $2.888 E-12$ & $1.733 \mathrm{E}-10$ & 8837052355 & 18945293.6 & $9923013.0 \mathrm{~B}$ & 158768067.4 & 635072175 \\
\hline $\mathrm{PIn+147}$ & 4440 & $1.64 \mathrm{E}+11$ & 1900000000 & 710 & $6.357 \mathrm{E}-34$ & $3.814 \mathrm{E}-32$ & 3701557779 & $7935575 . \overline{82}$ & $9.1482 \mathrm{E}-16$ & $1.46371 \mathrm{E} \cdot 14$ & $5.855 E-14$ \\
\hline CerPr-144 & 303 & $1.12 \mathrm{E}+10$ & $5.2 \mathrm{E}+11$ & 852 & $5.564 \mathrm{E}-40$ & $3.338 E-38$ & 252606308 & 541549.431 & $5.4643 \mathrm{E}-23$ & 8.74285E-22 & $3.497 \mathrm{E}-21$ \\
\hline Eu-154 & 225 & $8.33 E+09$ & $1.90 E+09$ & 710 & $6.357 \mathrm{E}-34$ & $3.814 E+32$ & 187578942 & 402140.667 & $4.6359 \mathrm{E}-17$ & $7.41744 E-16$ & $2.967 \mathrm{E}-15$ \\
\hline U-234 & 0.395 & $1.46 \mathrm{E}+07$ & $1.90 \mathrm{E}+09$ & 710 & $6.357 \mathrm{E}-34$ & $3.814 E-32$ & $329305.25 \overline{3}$ & 705.880281 & B.1386E-20 & $1,3021 \mathrm{TE}-18$ & $5.209 E-18$ \\
\hline $\mathrm{Cs}-134$ & 567 & $2.10 \mathrm{E}+10$ & 850000 & 292 & $2.88 B E-12$ & $1.733 \mathrm{E}-10$ & 472688933 & 1013324.48 & 530787.586 & 8492593.795 & 33970370 \\
\hline Pu-242 & 0.0566 & $209 \mathrm{E}+06$ & $1.90 \mathrm{E}+09$ & 710 & $6.357 \mathrm{E}-34$ & $3.814 \mathrm{E}-32$ & 47186.5248 & 101.160719 & $1.1662 \mathrm{E}-20$ & $1.8659 \mathrm{E}-19$ & $7464 E+19$ \\
\hline Sb/Tem-125 & 345.8 & $1.28 E+10$ & 262 & 207 & $1.083 \mathrm{E}-10$ & 6.497E-09 & 288297004 & 618064.844 & 12138284.3 & 194212375 & 776849384 \\
\hline Cd. $113 \mathrm{~m}$ & 4.04 & $1.49 E+08$ & 1000000000 & 710 & $6.357 \mathrm{E}-34$ & $3.814 \mathrm{E}-32$ & 3368084.11 & 7220.65908 & $8.324 \mathrm{E}-19$ & $1.33+94 \mathrm{E}-17$ & 5.327E-17 \\
\hline Sm-151 & 101 & $3.74 E+09$ & $5.2 E+11$ & 852 & $5.564 E-40$ & $3.338 \mathrm{E}-3 \mathrm{~B}$ & 84202102.6 & 180516.477 & $1.82+4 E-23$ & $2.91432 E-22$ & $1.166 \mathrm{E}-21$ \\
\hline Eu-155 & 65.4 & $2.42 E+09$ & $1.90 E+09$ & 710 & $6.357 E-34$ & $3.814 \mathrm{E}-32$ & 54522945.7 & 116898.887 & $1.3475 \mathrm{E}-17$ & $2.156 \mathrm{E}-16$ & $8.624 \mathrm{E}-16$ \\
\hline Co-60 & 9.74 & $3.60 E+O B$ & $1.90 E+09$ & 710 & $6.357 \mathrm{E} \cdot 34$ & $3.814 E-32$ & $8 \overline{120083.96}$ & 17408.2226 & $2.0068 E-18$ & 3.21093E-17 & 1.284E-16 \\
\hline EU-152 & 1.21 & $4.48 E+07$ & $1.005+09$ & 710 & 6.357E-34 & $3 . B 14 \mathrm{E}-32$ & 1008757.86 & 2162.62314 & $2.4931 \mathrm{E}-19$ & $3.98893 \mathrm{E}-18$ & 1.596E-17 \\
\hline $4-129$ & 1 & $3.70 E+07$ & $1.90 \mathrm{E}+07$ & 335 & $1.728 \mathrm{E}-13$ & $1.037 \mathrm{E}-11$ & $833 \overline{684.184}$ & 1787.29185 & 56.0029225 & 896.0459601 & 3584.1833 \\
\hline $\mathrm{Kr}-85$ & 772 & $2.86 \mathrm{E}+10$ & 32000 & 242 & $1.066 \mathrm{E}-10$ & $6.393 E-09$ & 643604180 & 1379789.31 & 26663709.3 & 426618967.6 & $1.706 E+09$ \\
\hline Fe- 55 & 21.8 & $8.07 \mathrm{E}+08$ & $1.80 \mathrm{E}+09$ & 710 & $6.357 \mathrm{E}-34$ & $3.814 E-32$ & 18174315.2 & 38962.9624 & 4.4917E-18 & $7.18667 \mathrm{E}-17$ & $2.875 E-16$ \\
\hline $\mathrm{N} \mathrm{N}-63$ & 3.11 & $1.15 \mathrm{E}+08$ & $1.90 \mathrm{E}+0 \mathrm{OS}$ & 710 & $6.357 \mathrm{E}-34$ & $3.814 \bar{E}-32$ & 2592757.81 & 5558.47766 & $6.4078 \mathrm{~B}-19$ & $1.02525 \mathrm{E}-17$ & $4.101 \mathrm{E}-17$ \\
\hline Tc-99 & 1.72 & $6.36 \mathrm{E}+07$ & 1900000000 & 710 & $6.357 E-34$ & $3.814 E-32$ & 1433936.8 & 3074.14189 & $3.5439 \bar{E}-19$ & $5.67022 \mathrm{E}-18$ & 2.268E-17 \\
\hline Se-79 & 1 & $3.70 E+07$ & 1.2 & 200 & $1.301 \mathrm{E}-12$ & $7.805 E-11$ & 833684.184 & 1787.29185 & 421.668855 & 6746.68566 & 26986779 \\
\hline $\mathrm{H}-3$ & 45.43 & $1.68 \mathrm{E}+09$ & & & & & 37674047.2 & & $1.3635 E+11$ & $5.45386 E+11$ & $1.001 E+12$ \\
\hline$c-14$ & 0,443 & $1.64 \mathrm{E}+07$ & 32000 & 242 & $1.066 \mathrm{E} \cdot 10$ & $6.393 \mathrm{E}-09$ & 369322.094 & 791.77029 & 15300.5482 & 244808.5527 & 979234.06 \\
\hline Assume ambient & air pulled & to $\mathrm{MCO}$ & Oxidatio & Rates & & & & & & & \\
\hline Temp ${ }^{\circ} \mathrm{C}$ & & & $\mathrm{mg} \mathrm{O}_{2} / \mathrm{cm}^{2} / \mathrm{sec}$ & $g \mathrm{U} / \mathrm{cm}^{2} / \mathrm{sec}$ & & & & & & & \\
\hline $600^{\circ} \mathrm{C}$ oxidation & 873.16 & & 3029513275 & 0.022532 & & & & & & & \\
\hline
\end{tabular}


WHC-SD-SNF-TI-033

Revision 0

\subsection{CASE 2}

Table 4 gives the integrated release of fission products in $\mathrm{Bq} / \mathrm{cm}^{2}$ for simulated hot vacuum drying conditions $\left(300^{\circ} \mathrm{C}\right.$ and at 5 torr water pressure).

Table 4. Fission Product Release During Hot Vacuum Drying.

\begin{tabular}{|c|c|c|c|c|c|c|c|c|c|c|c|}
\hline \multirow{3}{*}{ Sotope } & \multirow{3}{*}{ cinis } & \multicolumn{2}{|c|}{ Kako'Explakt] } & \multicolumn{2}{|c|}{ Input Tem300 ' C } & \multirow{3}{*}{$k, 1 / 4$} & \multirow{3}{*}{ Mro } & \multirow{3}{*}{$\begin{array}{c}\text { Mr } \\
\text { eotent }\end{array}$} & \multirow{2}{*}{\multicolumn{3}{|c|}{ 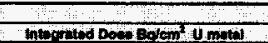 }} \\
\hline & & Bqhou & Ko, thmin: & Q,kilmol & k,Umin & & & & & & \\
\hline & & & & & & & & & 5.000 & 1000 & 20000 \\
\hline Pu-241 & 9450 & $3.4965 E+11 !$ & $1.90 \mathrm{E}+09$ & 710 & $3.72 E-56$ & $2.23 E-54$ & 2287.342086 & 16869908 & $1.68949 \mathrm{E}-43$ & $1.07 E-42$ & $3.20589 \mathrm{E}-42$ \\
\hline $\mathrm{am}-241$ & 142 & 5254000000 & $1.90 \mathrm{E}+09$ & 710 & $3.72 E-56$ & $2.23 E-54$ & 34.37064298 & 253795.443 & $2.5387 E-45$ & $1.61 E-44$ & $4.81731 \mathrm{E}-44$ \\
\hline Pu-239 & 139 & 5143000000 & $1.90 \mathrm{E}+09$ & 710 & $3.72 \mathrm{E}-55$ & $2.23 E-54$ & $33.644502 E 3$ & 242433.567 & $2.48506 E-45$ & $1.58 E-44$ & 4.71554E-44 \\
\hline Pu-238 & 116 & 4292000000 & $1.90 \mathrm{E}+09$ & 710 & $372 \mathrm{E}-56$ & $2.23 E-54$ & 2807742666 & 207325.855 & $2.07387 E-45$ & $1.32 E-44$ & $3.93527 \mathrm{E}=44$ \\
\hline Pu-240 & 95.2 & $3522400000^{\circ}$ & $1.90 E+09$ & 710 & $3.72 \mathrm{E}-56$ & $2.23 E-54$ & 23.0429536 & 170150.184 & $1.702 E-45$ & $1.08 \mathrm{E}-4$ & $3.22963 \mathrm{E}-44$ \\
\hline $\mathrm{SeN} \times 90$ & 8480 & $3.1376 \mathrm{E}+11$ & 1.2 & 200 & $7.11 E-19$ & $4.26 E-17$ & 2052.556708 & 15156234.9 & $2.89342 E-06$ & $1.84 E-05$ & $5.49041 E-05$ \\
\hline Cm-244 & 26.8 & 991600000 & $1.90 E+09$ & 710 & $3.72 E-56$ & $2.23 E-54$ & 6.466853745 & 47099.4216 & $4.79+34 E-A 6$ & $3.04 E-45$ & $9.09182 E-45$ \\
\hline Rw/Rh m-106 & 2430 & $8.991 \mathrm{E}+10$ & 1900000000 & 710 & $3.72 E-56$ & 2.23E-54 & 588.1736792 & 4343119.2 & $4.34439 E-44$ & $2.76 E-43$ & 8.24371E-43 \\
\hline 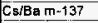 & 10600 & 3.922E+11 & 850000 & 292 & $2.08 E \cdot 21$ & $1.25 E-19$ & 2565.695084 & 18945293.6 & $1.05653 \mathrm{E}-0 \mathrm{~A}$ & 6.71E-08 & $2.00483 \mathrm{E}-07$ \\
\hline$\overline{P m-147}$ & 4440 & $9.6428 \mathrm{E}+11$ & 1900000000 & 710 & $3.72 E-56$ & $223 E-54$ & 107468771 & $7935575 . \overline{8}$ & $7.9379 E-44$ & $5.04 E-43$ & $1.50626 \mathrm{E}-42$ \\
\hline CeIPr-144 & 303 & $1.1211 \mathrm{E}+10$ & 5.2E+11 & 852 & $1.17 E-66$ & $6.99 E-65$ & 73.34017481 & 541549.431 & $1.6961 \mathrm{E}-55$ & $1.08 \mathrm{E}-54$ & $3.21843 \mathrm{E}-54$ \\
\hline$E \mathrm{u}-154$ & 225 & 8325000000 & $1.90 E+09$ & 710 & $3.72 \mathrm{E}-56$ & $2.23 E-54$ & 54.46052585 & 402140.667 & $4.02258 E-45$ & $2.56 E-44$ & $7.63306 E-44$ \\
\hline 0.234 & 0395 & 14615000 & $1.90 E+09$ & 710 & $3.72 E-56$ & 2.23E-54 & 0.095608479 & 705.980281 & $706187 \mathrm{E}-48$ & $4.49 E-47$ & $1.34003 \mathrm{E}-46$ \\
\hline Cs-134 & 567 & $2.0979 \mathrm{E}+10$ & 850000 & 292 & $2.08 \mathrm{E}-21$ & 1.25E-19 & 137.2405251 & 1013394.48 & $5.65146 \mathrm{E}-10$ & 3.59E-09 & $1.07239 \mathrm{E}-0 \mathrm{~B}$ \\
\hline PU-242 & 0.0566 & 2094200 & $1.90 E+09$ & 710 & $3.72 \mathrm{E}-56$ & $2.23 E-54$ & 0.013695648 & 101.160719 & $1.0119 E-48$ & $5.43 \mathrm{E}-48$ & $1.92014 E-47$ \\
\hline $\mathrm{S} \mathrm{S} / \mathrm{T} \mathrm{T}-12 \mathrm{~S}$ & 345.8 & $1.2795 E+10$ & 262 & 207 & $3.57 \mathrm{E}-17$ & $2.14 E-15$ & 8370239378 & 618064.844 & $5.92938 \mathrm{E}-06$ & $3.77 \mathrm{E}-05$ & 0.000112513 \\
\hline $\mathrm{Co}-113 \mathrm{~m}$ & 4.04 & 149480000 & 1900000000 & 710 & $3.72 E-56$ & $2.23 \mathrm{E}-54$ & 0.977868997 & 7220.65908 & $7.22277 \mathrm{E}-47$ & $4.59 \mathrm{E}-46$ & $1.37056 \mathrm{E}-45$ \\
\hline $5 m-151$ & 101 & 3737000000 & $5.2 \mathrm{E}+11$ & BS2 & $1.17 E-56$ & $6.99 \mathrm{E}-65$ & 24,44672494 & 180516.477 & $5.65366 \mathrm{E}-56$ & 3.59E-55 & $1.07281 \mathrm{E}-5 \mathrm{~A}$ \\
\hline ËL-155 & 654 & 2419800000 & $190 E+09$ & $7 \times 0$ & $3.72 \mathrm{E}-56$ & $2.23 E \cdot 54$ & 15.82985951 & 116888.897 & 1.16923E-45 & $7.43 E-45$ & $2.21868 \mathrm{E}-44$ \\
\hline Co-60 & 9.74 & 360380000 & $1.90 \mathrm{E}+09$ & 740 & $3.72 \mathrm{E}-56$ & $2.23 \mathrm{E} \cdot 54$ & 2.357535652 & 17408.2226 & 1.74133E- -66 & $1.11 \mathrm{E}-45$ & $3.30427 \mathrm{E}-45$ \\
\hline Eu-152 & 1.21 & 44770000 & $1.90 \mathrm{E}+09$ & 710 & $3.72 E-56$ & 2.23E- 54 & 0.292876606 & 2162.62314 & $2.16326 \mathrm{E}-47$ & $1.37 E-46$ & $4.10489 E-46$ \\
\hline 1.129 & 1 & 37000000 & $1.90 \mathrm{E}+07$ & 335 & $5.59 E \cdot 24$ & $336 \mathrm{E} \cdot 22$ & 0.242046782 & 1787.29185 & $2.68527 \mathrm{E}-15$ & $1.71 \mathrm{E}-14$ & $5,09544 E-14$ \\
\hline$k_{r-85}$ & 772 & $2.8564 \mathrm{E}+10$ & 32000 & 242 & $2.82 E-18$ & $1.69 \mathrm{E}-16$ & 186.8601154 & 1379789.31 & $1.04427 E-06$ & $0.63 \mathrm{E}-06$ & $1.98156 E .05$ \\
\hline $\mathrm{Fe}-55$ & 218 & $806600000_{1}$ & $1.90 E+09$ & 710 & $3.72 \mathrm{E}-56$ & $2.23 E-54$ & 5.276619838 & 38962.9624 & $3.89744 E-46$ & $248 E-45$ & $739559 \mathrm{E}-45$ \\
\hline Ni.63 & 3.11 & 115070000 & $190 \mathrm{E}+09$ & 710 & $3.72 E-56$ & $2.23 E-54$ & 0.752765491 & 5558.47766 & $5.5601 E-47$ & $3.53 E-46$ & 1.05506E-45 \\
\hline $\mathrm{T}=-99$ & 1.72 & 63640000 & 1900000000 & 710 & $3.72 \mathrm{E}-56$ & $2.23 E-54$ & 0.416320464 & 3074,14199 & $307504 E-47$ & $1.95 \mathrm{E}-46$ & $5.23505 E-46$ \\
\hline Se-79 & 1 & 37000000 & 1.2 & 200 & $7.11 \mathrm{E}-19$ & $4 \overline{26 E-17}$ & 0.242046782 & 1787.29185 & $341205 E-10$ & $2.17 E-09$ & $6.47454 \mathrm{E}-09$ \\
\hline $\mathrm{H}-3$ & 45.43 & 1680900000 & & & & & 10.99611987 & & 39586.03152 & 158344.1 & 316688.2522 \\
\hline \multirow[t]{2}{*}{$c-14$} & 0.443 & 16391000 & & & & & 0.107226724 & & 386.0162072 & 1544.065 & 3088.129658 \\
\hline & & & & & & & & & & & \\
\hline \multirow[t]{2}{*}{ HVD at $300^{\circ} \mathrm{C}$} & & & & \multicolumn{2}{|c|}{ Oxication Rate } & & & & & & \\
\hline & Temp ${ }^{2} K$ & & & $\mathrm{mg} \mathrm{O}_{2} / \mathrm{cm}^{2} / 5$ & $\mathrm{~kg} \mathrm{U} / \mathrm{cm}^{2} / \mathrm{sec}$ & & & & & & \\
\hline $50^{\circ} \mathrm{C}$ & 573.16 & & 0.664473684 & 0.00087957 & $6.542 \mathrm{E}-09$ & & & & & & \\
\hline & & & & & & & & & & & \\
\hline
\end{tabular}


WHC-SD-SNF-TI-033

Revision 0

\subsection{CASE 3}

Table 5 gives the integrated release of fission products in $\mathrm{Bq} / \mathrm{cm}^{2}$ for simulated cold vacuum drying conditions $\left(50^{\circ} \mathrm{C}\right.$ and at 5 torr water pressure).

Table 5. Fission Product Release During Cold Vacuum Drying.

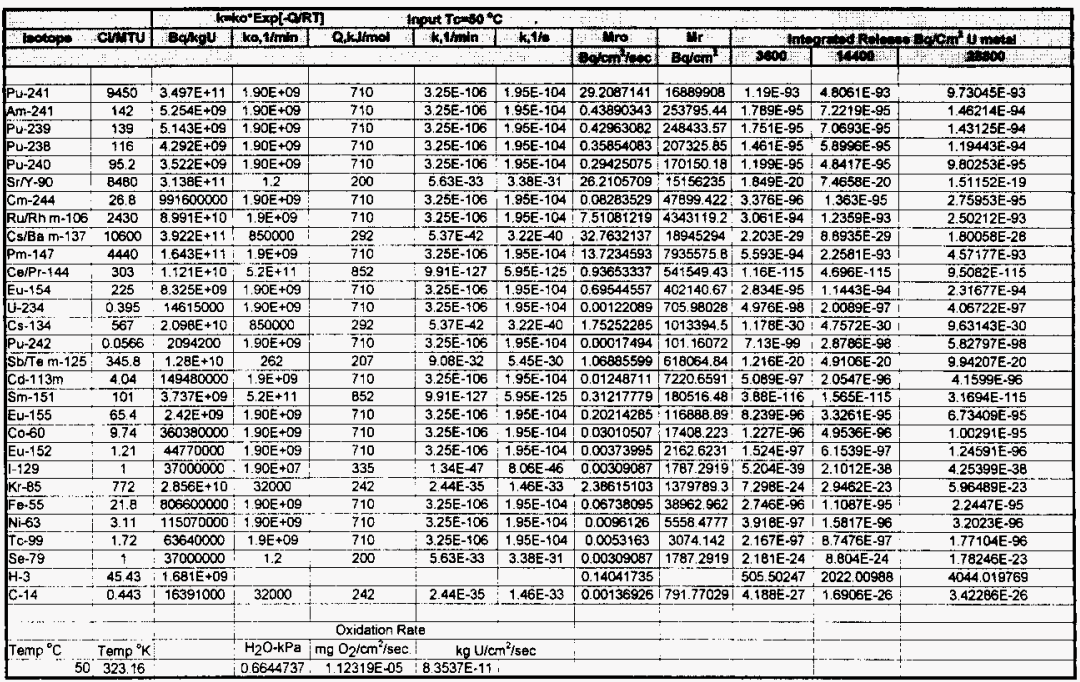


WHC-SD-SNF-TI-033

Revision 0

\subsection{CASE 4}

Table 6 gives the integrated release of fission products in $\mathrm{Bq} / \mathrm{cm}^{2}$ for simulated hot conditioning conditions ( $150^{\circ} \mathrm{C}$ and at $2 \%$ oxygen in an argon atmosphere).

Table 6. Fission Product Release During Hot Conditioning.

\begin{tabular}{|c|c|c|c|c|c|c|c|c|c|c|c|}
\hline & & & KOEXPLOI & & Putcelt & $c$ & & & & & \\
\hline Fotope & CHMTI & Eglkou & to, 1/min & Q kJ/mol & 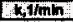 & $k, 1 / 2$ & No & Mr & 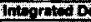 & Bg pos: & \\
\hline & & & & & $\begin{array}{c}0 \\
\times \cdots \cdots \\
\end{array}$ & & Bden ${ }^{2}$ ares & Boten: & 3600 & 1450 & etoes \\
\hline$P_{U-241}$ & 9450 & $3.4965 E+11$ & $1.9 E+09$ & 710 & $4.30 \mathrm{E}-79$ & $.58 \mathrm{E}-77$ & 251532.811 & 16889908 & $1.5691 E-66$ & $6.2765 E-66$ & $1.2553 \mathrm{E}-65$ \\
\hline $\mathrm{Am}-241$ & 142 & 5254000000 & $1.9 E+09$ & 710 & $4.30 E-79$ & 2.58E-77 & 3779.64648 & 253795.44 & $2.3578 \mathrm{E}-68$ & $9.4313 E-68$ & $1.88628 \mathrm{E}-67$ \\
\hline$P_{4-239}$ & 139 & 5143000000 & 1.9E+09 & 710 & $4.30 \bar{\varepsilon}+79$ & $2.58 E+77$ & 3609.79479 & 248433.57 & $2,308 E-68$ & $9.2321 \mathrm{E}-68$ & 1.84641E-67 \\
\hline$P 4=238$ & 116 & 4292000000 & $1.9 E+09$ & 710 & $4.30 \mathrm{E}-79$ & $2.58 \mathrm{E}-77$ & 3087.59853 & 207325.85 & $1.9261 \mathrm{E}-6 \mathrm{~B}$ & $7.7045 \mathrm{E}-68$ & $1.54088 E-67$ \\
\hline$P 山-240$ & 95.2 & 3522400000 & $1.9 \mathrm{E}+09$ & 710 & $4.30 E-78$ & $2.5 \mathrm{BE}-\overline{7} 7$ & 2533.98017 & 170150.18 & 1.5807E-6B & $6.323 \mathrm{E}-6 \mathrm{~B}$ & $1.26459 E-67$ \\
\hline$S \pi / \gamma-80$ & 8480 & $3.1376 \mathrm{E}+11$ & 1.2 & 200 & $2.46 \mathrm{E}-25$ & $1.47 \mathrm{E}-23$ & 225714.1 & 15156235 & 8.0435E-13 & $3.2174 \mathrm{E}-12$ & $6.43478 E-12$ \\
\hline $\mathrm{Cm}-244$ & 26.8 & 901600000 & $1.9 E+09$ & 710 & $4.30 \mathrm{E} \cdot 79$ & $2.58 \mathrm{E}-77$ & 713.34173 & 47899.422 & $4.45 \mathrm{E}-69$ & $1.78 \mathrm{E}-6 \mathrm{~B}$ & $3.55999 E-68$ \\
\hline $\mathrm{R} u / \mathrm{Rh} \mathrm{m}-106$ & 2430 & 89910000000 & 1.9E+09 & 710 & $4.30 \mathrm{E}-79$ & $2.58 E-77$ & 64679.8658 & 4343149.2 & 4.0349E-67 & $1.614 \mathrm{E}-66$ & $3.2279 E-66$ \\
\hline $\mathrm{Cs} / \mathrm{Ba} \mathrm{m}-137$ & 10600 & $3.922 \mathrm{E}+11$ & 850000 & 292 & $765 \mathrm{E}-31$ & $4.59 \mathrm{E}-29$ & 282142.624 & 18945294 & 3.1315E-18 & $1.2526 \mathrm{E}-17$ & $2.50519 \mathrm{E}-17$ \\
\hline $\mathrm{Pm}-147$ & 4440 & 1.6428E+11 & $1.9 E+09$ & 710 & $4.30 \mathrm{E}-79$ & 2.58E-77 & 118180.496 & 7935575.8 & $7.3724 \mathrm{E}-67$ & $2.8489 \mathrm{E}-68$ & $5.89789 E-66$ \\
\hline CE & 303 & 11211000000 & $5.2 \mathrm{E}+11$ & 852 & $3.48 \mathrm{E}-94$ & $2.09 \mathrm{E}-92$ & 8065.0203 & 541549.43 & $4.0727 \mathrm{E}-83$ & $1.6291 \mathrm{E}-82$ & $3.25815 E-82$ \\
\hline Eu-154 & 225 & 8325000000 & $1.9 E+09$ & 710 & $4.30 E-79$ & $2.58 \mathrm{E}-77$ & 5988.87646 & 402140.67 & $3.736 \mathrm{E}-68$ & $1.4944 E-67$ & $2.9888 E-67$ \\
\hline $0-234$ & 0.395 & 14615000 & $1.9 E+09$ & 710 & $4.30 \mathrm{E} \cdot 79$ & $2.58 \mathrm{E}-7 \overline{7}$ & 10.5138053 & 705.98028 & $6.55 \mathrm{~B} 8 \mathrm{E}-71$ & $2.6235 \mathrm{E}-70$ & $5.24701 \mathrm{E}-70$ \\
\hline Cs-134 & 567 & 20979000000 & 850000 & 292 & $7.65 \mathrm{E}-31$ & $4.59 \mathrm{E}-29$ & 15091.9687 & 1013394.5 & $1 . 6 7 5 1 \longdiv { \mathrm { E } - 1 9 }$ & $6.7002 \mathrm{E}-19$ & $1.34004 \mathrm{E}-18$ \\
\hline$P_{U-242}$ & 0.0566 & 2094200 & $1.9 \mathrm{E}+09$ & 710 & $4.30 \mathrm{E}-79$ & $2.58 E-77$ & 1,50653515 & 101.16072 & 9.3981E-72 & $3.7593 \mathrm{E}-71$ & 7.51861E-71 \\
\hline Sb/Te m-125 & 345.8 & 12795000000 & 262 & 207 & $7.34 \mathrm{E}-24$ & $4.40 E-22$ & 9204.52544 & 618064.84 & $9.7926 \mathrm{E}-13$ & $3.917 \mathrm{E}-12$ & $7.6341 E-12$ \\
\hline Cd-113m & 4.04 & 149480000 & $1.9 \mathrm{E}+09$ & 710 & 4.30E-79 & $2.58 \mathrm{E}-77$ & 107.533604 & 7220.6591 & $6.7082 \mathrm{E}-70$ & $2.6833 E-69$ & $5,36655 \mathrm{E}-69$ \\
\hline$S m-151$ & 101 & 3737000000 & $5.2 E+17$ & 852 & $348 E-94$ & 2.09E-92 & 2688.3401 & 480516.48 & $1.3576 \mathrm{E}-83$ & $5.4302 \mathrm{E}-63$ & 1.08605E-82 \\
\hline Eu-155 & 65.4 & 2419800000 & $1.9 \mathrm{E}+00$ & 710 & $4.30 E-79$ & $2.58 \mathrm{E}-77$ & 1740.76676 & 116888.89 & 1.0859E-68 & $4.3437 E-68$ & B.68744E-6B \\
\hline Co-60 & 9.74 & 360380000 & $1.9 E+09$ & 710 & $430 \mathrm{E}-79$ & $2.58+E-77$ & 250.251808 & 17408.223 & $1.6173 \mathrm{E}-69$ & $6.4601 \mathrm{E}-69$ & 1.29382E-6B \\
\hline Eu-152 & 1.21 & 44770000 & $1.9 E+09$ & 710 & $4.30 \mathrm{E}-79$ & $2.58 \mathrm{E}-77$ & 32.2068468 & 2162.6231 & $2,0091 \mathrm{E}-70$ & $8.0365 E-70$ & $1.60731 \mathrm{E}-69$ \\
\hline $1-129$ & 1 & 37000000 & 19000000 & 335 & $8.41 E-35$ & $5.05 E-33$ & 26.6172287 & 1787.2919 & $3.2486 \mathrm{E}-26$ & $1.2994 \mathrm{E}-25$ & $2.59886 \mathrm{E}-25$ \\
\hline $\mathrm{Kr}-85$ & 772 & 28564000000 & 32000 & 242 & $4.28 E-26$ & $2.57 E-24$ & 20548.5006 & 1379788.3 & 1.2764E-14 & $5.1056 \mathrm{E}-14$ & $1.02113 \mathrm{E} \cdot 13$ \\
\hline $\mathrm{Fe} .55$ & 218 & 806600000 & $1.9 E+09$ & 710 & 4. 30E-79 & $2.581 \mathrm{E}-77$ & 580.255586 & 38962.962 & $3.6498 \mathrm{E}-69$ & $1.4479 \mathrm{E}-68$ & $2 \overline{89581 E-68}$ \\
\hline $\mathrm{Ni}-63$ & 3.11 & 115070000 & $1.9 \mathrm{E}+09$ & 710 & $4.30 \equiv-79$ & $2.581 \mathrm{E}-77$ & 82.7795813 & 5558.4777 & $5.164 \mathrm{E}-70$ & $2.0656 \mathrm{E}-69$ & 4. $13118 \mathrm{E}-69$ \\
\hline$T c-99$ & 1.72 & 63640000 & $1.9 E+09$ & 710 & 4.30E.79 & $2.58 \mathrm{E}-77$ & 45.7816334 & 3074.142 & $2.856 \mathrm{E}-70$ & $1.1424 \mathrm{E}-69$ & 2.28477 E- 69 \\
\hline Se-79 & 1 & 37000000 & 1.2 & 200 & $2.46 E-25$ & 1.47E-23 & 26.6172287 & 1787.2919 & $94852 \mathrm{E}-17$ & $3.794+E-16$ & $7.58819 E-16$ \\
\hline $\mathrm{H}-3$ & 45.43 & 1680900000 & & & & & $196.6809 \mathrm{e} 3$ & & 708051.539 & $2832206 . \overline{15}$ & 5664412.31 \\
\hline C-14 & 0.443 & 16391000 & 32000 & 242 & $4,28 \mathrm{E}-26$ & $2.57 E-24$ & 11.7914323 & 791.77029 & $7.3245 \mathrm{E}-18$ & $2.9298 \mathrm{E}-17$ & $5.8585 B \mathrm{BE}-17$ \\
\hline & & & & & & & & & & & \\
\hline & & & & & Rate & & & & & & \\
\hline $\operatorname{Temp}{ }^{\circ} \mathrm{C}$ & Temp ${ }^{\circ} \mathrm{K}$ & & $1 \cdots=0<1$ & $\mathrm{~kg}$ U/sec(air) & $\mathrm{U} / \mathrm{sec}(2 \%$ & & & & & & \\
\hline 150 & 42316 & & 0.096724 & 7.1938E-07 & $1.17 \mathrm{E} .07$ & & & & & & \\
\hline
\end{tabular}


WHC-SD-SNF-TI-033

Revision 0

\subsection{CASE 5}

Table 7 gives the integrated release of fission products in $\mathrm{Bq} / \mathrm{cm}^{2}$ for simulated long-term storage conditions $\left(50^{\circ} \mathrm{C}\right.$ and at 0 torr water and oxygen pressures).

Table 7. Fission Product Release During Long Term Storage.

\begin{tabular}{|c|c|c|c|c|c|c|c|c|c|}
\hline \multicolumn{10}{|c|}{ Funo'ExpLORI } \\
\hline Lotope & chartu & Eqhgu : & wo, thain & Qtitimol & k, $1 / m$ ln & k,16: & Aro & mer & 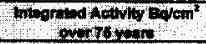 \\
\hline$\therefore \quad \because$ & & & & & $\therefore \quad \cdots$ & & Bofcmbec & $40 \mathrm{~cm}^{2}$ & Bronorobos \\
\hline$P u-241$ & 9450 & $3.4965 \mathrm{E}+11$ & $1.90 \mathrm{E}+09$ & 710 & $7.86 \mathrm{E}-70$ & $4.71 E-68$ & 0 & 16889908 & $1.88453 E-51$ \\
\hline$A m-241$ & 142 & 5254000000 & $1.90 \mathrm{E}+09$ & 710 & 7.86E-70 & $4.74 E-68$ & 0 & 253795.443 & $2.83178 \mathrm{E}-53$ \\
\hline Pu-239 & 139 & 5143000000 & $1.90 \mathrm{E}+09$ & 710 & $7.86 \mathrm{E}-70$ & $4.71 \mathrm{E}-68$ & 0 & 248433.567 & $2.77196 \mathrm{E}-53$ \\
\hline Pu-238 & 116 & 4292000000 & $1.90 E+09$ & 710 & $7.86 \mathrm{E}-70$ & $4.71 E-68$ & 0 & 207325.855 & $2.31329 E-53$ \\
\hline$P u-240$ & 95.2 & 3522400000 & $1.90 \mathrm{E}+09$ & 710 & $7.86 \mathrm{E}-70$ & $471 \mathrm{E}-6 \mathrm{~B}$ & 0 & 170150.184 & $1.89849 E-53$ \\
\hline $\mathrm{Sr} / \mathrm{r}-90$ & 8480 & $3.1376 \mathrm{E}+11$ & 1.2 & 200 & $9.98 \mathrm{E}-23$ & $5.99 E-21$ & 0 & 15156234.9 & 0.000214898 \\
\hline $\mathrm{Cm}-244$ & 26.8 & 991600000 & $1.90 \mathrm{E}+09$ & 710 & $7.86 \mathrm{E}-70$ & $4.71 E-68$ & 0 & 47899.4216 & $5.3445 E-54$ \\
\hline Ru/Rh Tr-106 & 2430 & $8.991 E+10$ & 1900000000 & 710 & $7.86 \mathrm{E}-70$ & $4.71 E-68$ & 0 & 4343119.2 & 4.84594E-52 \\
\hline Cs/Ba m-137 & 10600 & $3.922 \mathrm{E}+11$ & 850000 & 292 & $4.93 \mathrm{E}-27$ & $2.96 \mathrm{E}-25$ & 0 & 18945293.6 & $1.32631 \mathrm{E}-0 \mathrm{~B}$ \\
\hline$P m-147$ & 4440 & $1.6428 \mathrm{E}+11$ & 1900000000 & 710 & $7.86 \mathrm{E}-70$ & 4.71E-68 & 0 & 7935575.82 & $8.85431 \mathrm{E}-52$ \\
\hline Ce/Pr- 144 & 303 & 1.1211E +10 & $5.2 \mathrm{E}+11$ & 852 & 4.53E- 83 & $2 . \overline{2} \overline{\mathrm{E}}-81$ & 0 & 541549.431 & $3.48149 \mathrm{E}-66$ \\
\hline Eu-154 & 225 & 8325000000 & $1.90 \mathrm{E}+09$ & 710 & $7.86 \mathrm{E}-70$ & 4.71E-68 & 0 & 402140.667 & $4.48698 \mathrm{E}-53$ \\
\hline 0.234 & 0.395 & 14615000 & $1.90 E+09$ & 710 & $7.86 \mathrm{E}-70$ & 4.71E-68 & 0 & 705.980281 & $7.87715 \mathrm{E}-56$ \\
\hline Cs-134 & 567 & $2.0979 \mathrm{E}+10$ & 850000 & 292 & $4.93 \mathrm{E}-27$ & 2.96E-25 & 0 & 1013394,48 & $7.09451 \mathrm{E}-10$ \\
\hline$P \bigsqcup-242$ & 0.0566 & 2094200 & $1.90 E+09$ & 710 & $7.86 \mathrm{E}-70$ & 4.71E-68 & 0 & 101.160719 & $1.12873 \mathrm{E}-56$ \\
\hline $\mathrm{Sb} / \mathrm{Te} \mathrm{m}-125$ & 345.8 & 1.2795E+10 & 262 & 207 & $3.68 \mathrm{E}-21$ & 2.21E-19 & 0 & 618054.844 & 0.000322849 \\
\hline Cd-113m & 4.04 & $149480000^{\circ}$ & 1900000000 & 710 & $7.86 \mathrm{E}-70$ & $4.71 E-68$ & 0 & 7220.65908 & $8.05663 \mathrm{E}-55$ \\
\hline $5 \mathrm{~m}-151$ & 101 & 3737000000 & $5.2 E+11$ & 852 & 4.53E-83 & $2.72 E-81$ & 0 & 180516.477 & 1.1605E-66 \\
\hline Eu-155 & 65.4 & 2419800000 & $1.90 \mathrm{E}+09$ & 710 & 7.86E-70 & $4.71 \mathrm{E}-68$ & 0 & 116888.887 & $1.30422 \mathrm{E}-53$ \\
\hline Co-60 & 9.74 & 360380000 & $1.90 \mathrm{E}+00$ & 710 & $7.86 E-70$ & $4.7142 \mathrm{E}-68$ & 0 & 17408.2226 & $1.94237 \mathrm{E}-54$ \\
\hline Eu-152 & 1.21 & 44770000 & $1.90 E+09$ & 710 & $7.86 \mathrm{E}-70$ & $471 \mathrm{E}-68$ & 0 & 2162.62314 & $2413 E-55$ \\
\hline $1-129$ & 1 & 37000000 & $1.90 E+07$ & 335 & $1.97 \mathrm{E}-30$ & $1.18 \mathrm{E}-28$ & 0 & $17 \overline{87.29185}$ & $5.00616 E-16$ \\
\hline $\mathrm{Kr}=\mathrm{B5}$ & 772 & $2.8564 E+10$ & 32000 & 242 & $6.15 \mathrm{E}-23$ & $3.69 \mathrm{E}-21$ & 0 & 1379789.31 & 1.20407E-05 \\
\hline Fo-55 & 21.8 & 806600000 & $1.90 E+09$ & 710 & $7.86 \mathrm{E}-70$ & $4.7142 E-68$ & 0 & 38962.9624 & $4.34739 \mathrm{E}-54$ \\
\hline $\mathrm{Ni}-63$ & 3.11 & 115070000 & $1.90 \mathrm{E}+09$ & 710 & $7.86 \mathrm{E}-70$ & $4.7142 E-68$ & 0 & 5558,47766 & 6.20201E-55 \\
\hline Tc-99 & 1.72 & 63640000 & 1900000000 & $710^{\circ}$ & $7.86 \mathrm{E}-70$ & $4.71 E-68$ & 0 & 3074.14199 & 3.43005E-55 \\
\hline Se-79 & 1 & 37000000 & 1.2 & 200 & $9.98 \mathrm{E}-23$ & 5.99E-21 & 0 & 1787.29185 & $2.53417 \mathrm{E}-08$ \\
\hline $\mathrm{H}-3$ & 45.43 & 1680900000 & & & & & 0 & & 0 \\
\hline$C-14$ & 0.443 & 16391000 & 32000 & 242 & 6. $15 \mathrm{E}+23$ & $3.69 \mathrm{E}-21$ & $D$ & 791.77029 & 6.90937 E-09 \\
\hline & & & & & & & & & \\
\hline & & & & Oxidation $R$ & ate & & & & \\
\hline Temp ${ }^{\circ} \mathrm{C}$ & Temp ${ }^{\circ} \mathrm{K}$ & & $\mathrm{H}_{2} \mathrm{O}-\mathrm{kPa}$ & $\mathrm{gO}_{2} / \mathrm{cm}^{2} / \mathrm{sec}$ & $\mathrm{kg} U / \mathrm{cm}^{2} / \mathrm{sec}$ & & & & \\
\hline 200 & 473.16 & & 0.001 & unknown & unknown-assu & med o for calc & culational pur & ses & \\
\hline
\end{tabular}


WHC-SD-SNF-TI-033

Revision 0

This page intentionally left blank. 


\subsection{DISCUSSION OF RESULTS}

From Table 3, at $600^{\circ} \mathrm{C}$, a significant number of fission product isotopes are released within 8 hours. These include Strontium-90, Cesium-137, Cesium-134, Antimony-125, lodine125, Krypton-85, Selenium-79, Tritium-3, and Carbon-14. Due to the low diffusion rate constants at or below $300{ }^{\circ} \mathrm{C}$, all significant amounts of the fission products are retained within the uranium oxide, except for tritium.

The tritium release rate is directly related to the oxidation rate of the uranium metal. The highest release rate is observed for Case 1 involving the $600{ }^{\circ} \mathrm{C}$ uranium oxidation.

The radiological dose delivered to site workers and the general public from fission product release should be evaluated. The radiological dose from internal absorption and from submersion in a gaseous atmosphere is given in Table 8.

To build an entire dose model, in addition to the data in Table 8, other parameters must be known or postulated. Examples of such parameters are: the oxidation rate of the metal (controlled by temperature, surface area and reactive gases), the release fraction (i.e., the ratio of the amount of isotope that goes airborne versus the amount that is retained on the oxide or other surfaces), the concentration of the isotopes in the atmosphere at given locations, and the breathing rate of exposed personnel.

Factors affecting the isotope concentrations are: the dilution rate due to mixing with noncontaminated gases, the distance from the source, and the diffusion rates of gases and dust particles bearing the isotopes.

The dose model is, therefore, site-specific and cannot be postulated generally. Due to the lack of site-specific information at this time; such as, size of rooms or buildings, ventilation exchange rates, site wind velocities, and diffusion rates of gases and dust particles, a site-specific dose model will be postponed.

Table 8. Internal and External Radiation Dose Estimates (2 Sheets).

\begin{tabular}{|c|c|c||}
\hline Isotope & $\begin{array}{c}\text { Efrective Dose } \\
\text { Internal } \\
\text { Sv/Bq }\end{array}$ & $\begin{array}{c}\text { Conversion Factors } \\
\text { Submersion } \\
\text { Sv-m } / \mathbf{B q}-\mathbf{h r}\end{array}$ \\
\hline Pu-241 & $2.23 \mathrm{E}-06$ & $0.00 \mathrm{E}+00$ \\
\hline Am-241 & $1.20 \mathrm{E}-04$ & $0.00 \mathrm{E}+00$ \\
\hline Pu-239 & $1.16 \mathrm{E}-04$ & $0.00 \mathrm{E}+00$ \\
\hline Pu-238 & $1.06 \mathrm{E}-04$ & $0.00 \mathrm{E}+00$ \\
\hline Pu-240 & $1.16 \mathrm{E}-04$ & $0.00 \mathrm{E}+00$ \\
\hline
\end{tabular}


Table 8. Internal and External Radiation Dose Estimates (2 Sheets).

\begin{tabular}{|c|c|c|}
\hline Isotope & $\begin{array}{c}\text { Effective Dose } \\
\text { Internal } \\
\text { Sv/Bq }\end{array}$ & $\begin{array}{c}\text { Conversion Factors } \\
\text { Submersion } \\
\text { Sv-m } / \mathbf{B q}-\mathbf{h r}\end{array}$ \\
\hline $\mathrm{Sr} / \mathrm{Y}-90$ & $3.53 \mathrm{E}-07$ & $0.00 \mathrm{E}+00$ \\
\hline $\mathrm{Cm}-244$ & $6.70 \mathrm{E}-05$ & $0.00 \mathrm{E}+00$ \\
\hline $\mathrm{Ru} / \mathrm{Rh} \mathrm{m}-106$ & $1.29 \mathrm{E}-07$ & $0.00 \mathrm{E}+00$ \\
\hline $\mathrm{Cs} / \mathrm{Ba} \mathrm{m}-137$ & 8.63E-09 & $0.00 \mathrm{E}+00$ \\
\hline Pm- 147 & $1.06 \mathrm{E}-08$ & $0.00 \mathrm{E}+00$ \\
\hline $\mathrm{Ce} / \operatorname{Pr}-144$ & $1.01 \mathrm{E}-07$ & $0.00 \mathrm{E}+00$ \\
\hline Eu-154 & $7.73 \mathrm{E}-08$ & $0.00 \mathrm{E}+00$ \\
\hline $\mathrm{U}-234$ & $3.58 \mathrm{E}-05$ & $0.00 \mathrm{E}+00$ \\
\hline Cs-134 & $1.25 \mathrm{E}-08$ & $0.00 \mathrm{E}+00$ \\
\hline $\mathrm{Pu}-242$ & $1.11 \mathrm{E}-04$ & $0.00 \mathrm{E}+00$ \\
\hline $\mathrm{Sb} / \mathrm{Te} \mathrm{m}-125$ & $5.27 \mathrm{E}-09$ & $0.00 \mathrm{E}+00$ \\
\hline $\mathrm{Cd}-113 \mathrm{~m}$ & 4.13E-07 & $0.00 \mathrm{E}+00$ \\
\hline Sm-15] & 8.10E-09 & $0.00 \mathrm{E}+00$ \\
\hline Eu- 155 & $1.12 \mathrm{E}-08$ & $0.00 \mathrm{E}+00$ \\
\hline $\mathrm{Co}-60$ & $5.91 \mathrm{E}-08$ & $0.00 \mathrm{E}+00$ \\
\hline Eu- 152 & $5.97 \mathrm{E}-08$ & $0.00 \mathrm{E}+00$ \\
\hline I-129 & $4.69 \mathrm{E}-08$ & $0.00 \mathrm{E}+00$ \\
\hline $\mathrm{Kr}-85$ & $0.00 E+00$ & $4.66 \mathrm{E}-11$ \\
\hline $\mathrm{Fe}-55$ & $7.26 \mathrm{E}-10$ & $0.00 \mathrm{E}+00$ \\
\hline $\mathrm{Ni}-63$ & $1.70 \mathrm{E}-09$ & $0.00 \mathrm{E}+00$ \\
\hline Tc-99 & $2.25 \mathrm{E}-09$ & $0.00 \mathrm{E}+00$ \\
\hline $\mathrm{Se}-79$ & $2.66 \mathrm{E}-09$ & $0.00 \mathrm{E}+00$ \\
\hline $\mathrm{H}-3$ & $1.73 \mathrm{E}-11$ & $1.19 \mathrm{E}-15$ \\
\hline $\mathrm{C}-14$ & $5.64 \mathrm{E}-10$ & $0.00 \mathrm{E}+00$ \\
\hline
\end{tabular}




\subsection{REFERENCES}

Kelly, J. L., 1984, Temperature Dependence of Fission Product Release Rates, Reynolds, A.B, McGown, M.E., Nuclear Science and Engineering.

NRC, 1981, Technical Basis for Estimating Fission Product Behavior During LWR Accidents, NUREG-0772, U. S. Nuclear Regulatory Commission, Washington, D.C.

Owczarski, P.C., 1996, Initial Models for Airborne Radionuclide Releases in an MCO, Informal Report, Science Applications International Corporation, Richland, Washington. 
WHC-SD-SNF-TI-033

Revision 0

This page intentionally left blank. 


\begin{tabular}{|c|c|c|c|c|c|}
\hline \multicolumn{6}{|c|}{ DISTRIBUTION SHEET } \\
\hline \multirow{2}{*}{$\begin{array}{l}\text { To } \\
\text { Distribution }\end{array}$} & \multirow{2}{*}{\multicolumn{3}{|c|}{$\begin{array}{l}\text { From } \\
\text { Plutonium Process Support } \\
\text { Laboratory/Thurman Cooper } \\
\end{array}$}} & \multicolumn{2}{|c|}{ Page 1 of 1} \\
\hline & & & & \multicolumn{2}{|c|}{ Date $07 / 30 / 96$} \\
\hline \multirow{2}{*}{\multicolumn{4}{|c|}{$\begin{array}{l}\text { Project Title/Work Order } \\
\text { SNFP Estimate of Volatile Fission Products Release From Multi-Canister } \\
\text { Overpacks, WHC-SD-SNF-TI-033. }\end{array}$}} & \multirow{2}{*}{\multicolumn{2}{|c|}{$\begin{array}{ll}\text { EDT No. } & 616633 \\
\text { ECN No. N/A }\end{array}$}} \\
\hline & & & & & \\
\hline 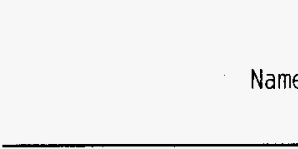 & MSIN & $\begin{array}{c}\text { Text } \\
\text { With } \\
\text { All } \\
\text { Attach. }\end{array}$ & Text Only & $\begin{array}{l}\text { Attach./ } \\
\text { Appendix } \\
\text { Only }\end{array}$ & $\begin{array}{c}\text { EDT/ECN } \\
\text { Onily }\end{array}$ \\
\hline $\begin{array}{l}\text { J Abrefah } \\
\text { GS Barney } \\
\text { GD Bazinet } \\
\text { LD Blackburn } \\
\text { TD Cooper } \\
\text { RG Cowan } \\
\text { ER Cramer } \\
\text { DR Duncan } \\
\text { FD Fisher } \\
\text { JR Frederickson } \\
\text { FJ Heard } \\
\text { JJ Irwin } \\
\text { A Johnson } \\
\text { AT Kee } \\
\text { CR Miska } \\
\text { SD Nunn } \\
\text { DM Ogden } \\
\text { PA Scott } \\
\text { PK Shen } \\
\text { DA Smith } \\
\text { TA Thornton } \\
\text { JC Wiborg } \\
\text { AM Zaman } \\
\text { Central Files (orig + 1) } \\
\text { SNF Project files }\end{array}$ & $\begin{array}{l}\text { P7-34 } \\
\text { T5-12 } \\
\text { B4-55 } \\
\text { R1-56 } \\
\text { T5-12 } \\
\text { R3-86 } \\
\text { H0-34 } \\
\text { R3-86 } \\
\text { T5-12 } \\
\text { R3-86 } \\
\text { H0-34 } \\
\text { H0-34 } \\
\text { K8-34 } \\
\text { R3-86 } \\
\text { R3-86 } \\
\text { S5-11 } \\
\text { H0-34 } \\
\text { R3-87 } \\
\text { HO- } 40 \\
\text { R3-11 } \\
\text { P7-18 } \\
\text { R3-86 } \\
\text { B1-10 } \\
\text { A3-88 } \\
\text { R3-11 }\end{array}$ & $\begin{array}{l}x \\
x \\
x \\
x \\
x \\
x \\
x \\
x \\
x \\
x \\
x \\
x \\
x \\
x \\
x \\
x \\
x \\
x \\
x \\
x \\
x \\
x \\
x \\
x \\
x\end{array}$ & & & . \\
\hline
\end{tabular}

